\title{
Influence de l'espèce et de la saison sur la dégradabilité de l'azote des fourrages verts dans le rumen
}

\author{
P Le Goffe 1, R Vérité 2, JL Peyraud 2 \\ Avec la collaboration technique de $\mathrm{H}$ Hétault, M Texier 2 \\ 1 ENSAR, 65, rue de Saint-Brieuc, 35042 Rennes Cedex; \\ 2 INRA, station de recherches sur la vache laitière, 35590 Saint-Gilles, France
}

(Reçu le 29 novembre 1991; accepté le 21 août 1992)

\begin{abstract}
Résumé - La dégradabilité de l'azote $(N)$ dans le rumen a été étudiée chez 9 plantes fourragères (4 ray-grass, 2 fétuques, 1 dactyle, 1 trèfle violet, 1 luzerne) au cours de 4 saisons (printemps précoce et tardif, juillet, septembre), en utilisant la méthode des sachets de nylon; 31 fourrages ont ainsi été récoltés à un stade précoce, entre le 3 avril et le 27 septembre, puis lyophilisés et broyés à la grille de $1,5 \mathrm{~mm}$. La dégradabilité in vitro de $\mathrm{N}$ (solubilité, fermentescibilité) et la composition chimique (matière sèche, $\mathrm{N}$ total, parois, $\mathrm{N}$ des parois) ont également été mesurées. La dégradabilité théorique de $\mathrm{N}$ en sachets de nylon (DTN avec un taux de passage de $0,06 \mathrm{~h}^{-1}$ ) varie peu selon l'espèce $(80-86 \%)$. Cependant, des différences notables existent quant à la valeur de la fraction rapidement dégradée (élevée chez la luzerne et faible au contraire chez le trèfle violet et le dactyle); ces écarts s'expliqueraient par les variations de la proportion d'azote sous forme non protéique, connues par ailleurs. La vitesse de dégradation de la fraction lentement dégradable est également faible chez le dactyle, probablement en relation avec des parois plus importantes et plus lignifiées, de sorte que sa DTN est la plus faible. DTN présente une évolution saisonnière : maximum au printemps $(87 \%)$, minimum en été $(80 \%)$ et remontée à l'automne $(82 \%)$; ces variations pourraient être en partie induites par la température moyenne pendant la pousse, qui agit notamment sur la lignification des parois.
\end{abstract}

fourrage vert / azote / rumen / dégradabilité / sachet

Summary - Influence of herbage species and harvest season on the rumen degradability of fresh forage nitrogen. Rumen degradability of feed nitrogen $(N)$ was studied in 9 herbage plants (4 rye-grasses, 2 fescues, 1 cocksfoot, 1 red clover, 1 luceme) at 4 seasons (early and late spring, summer (July), autumn (September)), using the nylon bag technique. Thirty-one forages were harvested at an early stage of maturity, between the April 3 and September 27, then freeze-dried and milled through a 1.5- $\mathrm{mm}$ screen. In vitro degradabilty (solubility and microbial fermentation) and chemical composition were also measured.

Theoretical rumen degradability (TDN) of feed $N$ in situ (TDN with outflow rate of $0.06 \mathrm{~h}^{-1}$ ) showed only limited vaniations between herbage species (86-80\%). However, noticeable differences were 
evidenced in the rapidly degradable fraction (higher with lucerne, and weaker with red clover and cocksfoot), which could have resulted from variations in the proportion of $N$ in non proteic form. The rate of degradation of cocksfoot $N$ was also low, in relation with higher and more lignified cell walls, inducing minimal TDN for cocksfoot. TDN exhibited a seasonal change, probably partially related to temperature which could have influenced cell wall lignification: TDN was maximum in spring (87\%), minimum in July (80\%) and increased again in September (82\%).

herbage / nitrogen / rumen / degradability / nylon bag

\section{INTRODUCTION}

L'influence de l'espèce et de la saison sur la composition chimique, la digestibilité et l'ingestibilité des fourrages verts, a été largement étudiée (Demarquilly et Weiss, 1970; Demarquilly et Jarrige, 1973; Demarquilly, 1982a). En revanche, l'effet de ces facteurs sur la dégradabilité des fourrages verts dans le rumen a fait l'objet d'expériences peu nombreuses, réalisées sur des populations limitées de fourrages, et relati /ement disparates quant à la nature du test de dégradabilité utilisé. Le manque d'informations concerne la dégradabilité de l'azote et la teneur en matières azotées non dégradables, mais également l'équilibre avec la dégradabilité des autres constituants, notamment les glucides.

L'objectif de notre travail était d'accroître les connaissances sur les variations de la dégradabilité ruminale des fourrages verts en fonction de l'espèce et de la saison, et de proposer des hypothèses d'explication de ces variations. Pour estimer la dégradation in vivo dans le rumen, nous avons retenu la méthode des sachets de nylon. Notre travail visait également à préciser les variations de la valeur azotée des fourrages verts, principalement dans sa fraction non dégradée dans le rumen, en vue d'optimiser leur utilisation zootechnique (choix de l'espèce, rationnement, etc).

\section{MATÉRIEL ET MÉTHODES}

\section{Matériel végétal}

Au Rheu (Île-et-Vilaine, France), 7 graminées et 2 légumineuses ont été récoltées à un stade précoce au cours de 4 périodes de l'année : au printemps en 1985 (avril-mai : $1^{\text {er }}$ cycle, uniquement feuillu chez les graminées) et en 1986 (mai-juin : $1^{\text {er }}$ cycle), en juillet 1985 (4e cycle pour les graminées, $2^{e}$ ou $3^{e}$ cycle pour les légumineuses) et en septembre 1985 (6 $6^{\ominus}$ cycle pour les graminées, $3^{e}$ ou $4^{e}$ cycle pour les légumineuses). Les espèces étudiées comprenaient des variétés de ray-grass italien (1), ray-grass hybride (1), ray-grass anglais (2), fétuque élevée (2), dactyle (1), trèfle violet (1) et luzerne (1). Toutes les espèces n'ont pu être récoltées à chaque saison. Au total, 31 fourrages ont été échantillonnés et leurs caractéristiques sont données dans le tableau 1.

La fertilisation azotée des graminées a été de $60 \mathrm{~kg} / \mathrm{ha}$ en fin d'hiver et après chaque coupe; les légumineuses n'ont pas reçu de fertilisation azotée. Après un déprimage (plante jeune uniquement feuillue), les graminées ont été exploitées en coupes fréquentes toutes les 4 à 5 semaines, dans le but de simuler le pâturage. Les légumineuses ont été exploitées à un rythme classique de fauche, au stade bourgeonnement. Pour caractériser les conditions de croissance, la température moyenne (des moyennes mini-maxi journalières) a été calculée sur les 28 j précédant la coupe, ou sur la période de croissance si elle est inférieure à $28 \mathrm{j}$.

Pour chacun des 31 fourrages, un échantillon moyen de $2 \mathrm{~kg}$ frais a été prélevé à partir de la récolte de 5 blocs (micro-parcelles de $5,4 \mathrm{~m}^{2}$ ). 
Tableau I. Date de récolte, rang de cycle, stade de développement et âge éventuel des $\mathbf{3 1}$ fourrages étudiés.

\begin{tabular}{|c|c|c|c|c|}
\hline Espèce et variété & Avril-mai 1985 & Mai-juin 1986 & Juillet 1985 & Septembre 1985 \\
\hline $\begin{array}{l}\text { Ray-grass italien } \\
\text { Adret }\end{array}$ & $\begin{array}{l}03 / 04 \\
\mathrm{C} 1^{1} \mathrm{FU}{ }^{2}\end{array}$ & & $\begin{array}{l}01 / 07 \\
\text { C4 RM 27j }\end{array}$ & \\
\hline $\begin{array}{l}\text { Ray-grass hybride } \\
\text { Augusta }\end{array}$ & $\begin{array}{l}\text { 03/04 } \\
\text { C1 FU }\end{array}$ & & $\begin{array}{l}01 / 07 \\
\text { C4 RM 23j }\end{array}$ & \\
\hline $\begin{array}{l}\text { Ray-grass anglais } \\
\text { Réveille }\end{array}$ & $\begin{array}{l}11 / 04 \\
\mathrm{C} 1 \mathrm{FU}\end{array}$ & & $\begin{array}{l}01-07 \\
\text { C4 RM 29j }\end{array}$ & $\begin{array}{l}04 / 09 \\
\text { C6 FU 37j }\end{array}$ \\
\hline $\begin{array}{l}\text { Ray-grass anglais } \\
\text { Vigor }\end{array}$ & $\begin{array}{l}26 / 04 \\
\text { C1 FU }\end{array}$ & $\begin{array}{l}12 / 05 \\
\text { C1 E10 26j }\end{array}$ & $\begin{array}{l}01 / 07 \\
\text { C4 RM } 32 j\end{array}$ & \\
\hline $\begin{array}{l}\text { Fétuque élevée } \\
\text { Clarine }\end{array}$ & $\begin{array}{l}17 / 04 \\
\text { C1 FU }\end{array}$ & $\begin{array}{l}07 / 05 \\
\text { C1 E10 35j }\end{array}$ & $\begin{array}{l}15 / 07 \\
\text { C4 FU 32j }\end{array}$ & $\begin{array}{l}16 / 09 \\
\text { C6 FU 34j }\end{array}$ \\
\hline $\begin{array}{l}\text { Fétuque élevée } \\
\text { Lubrette }\end{array}$ & $\begin{array}{l}17 / 04 \\
\text { C1 FU }\end{array}$ & $\begin{array}{l}07 / 05 \\
\text { C1 E10 35j }\end{array}$ & $\begin{array}{l}15 / 07 \\
\text { C4 FU 32j }\end{array}$ & $\begin{array}{l}16 / 09 \\
\text { C6 FU } 34 j\end{array}$ \\
\hline $\begin{array}{l}\text { Dactyle } \\
\text { Lutétia }\end{array}$ & $\begin{array}{l}26 / 04 \\
\text { C1 FU }\end{array}$ & $\begin{array}{l}07 / 05 \\
\text { C1 E10 35j }\end{array}$ & $\begin{array}{l}15 / 07 \\
\text { C4 FU 27j }\end{array}$ & $\begin{array}{l}16 / 09 \\
\text { C6 FU 34j }\end{array}$ \\
\hline $\begin{array}{l}\text { Trèfle violet } \\
\text { Tétri }\end{array}$ & $\begin{array}{l}22 / 05 \\
\text { C1 DB }\end{array}$ & $\begin{array}{l}02 / 06 \\
\text { C1 DB }\end{array}$ & $\begin{array}{l}15 / 07 \\
\text { C2 FL 55j }\end{array}$ & $\begin{array}{l}\text { 04/09 } \\
\text { C3 FL 52j }\end{array}$ \\
\hline Luzerne & $\begin{array}{l}14 / 05 \\
\text { C1 DB }\end{array}$ & $\begin{array}{l}13 / 05 \\
\mathrm{C} 1 \mathrm{DB}\end{array}$ & $\begin{array}{l}25 / 06 \\
\text { C2 BG 43j }\end{array}$ & $\begin{array}{l}27 / 09 \\
\text { C4 DF 60j }\end{array}$ \\
\hline Europe & & & $\begin{array}{l}30 / 07 \\
\text { C3 BG 36j }\end{array}$ & \\
\hline
\end{tabular}

$1 \mathrm{C1}$ : 1 er cycle; ${ }^{2} \mathrm{FU}$ : feuillu; $\mathrm{E} 10$ : épi à $10 \mathrm{~cm}$; RM : remontaison; DB : début bourgeonnement; BG : bourgeonnement; DF : début floraison; FL : floraison.

Les échantillons ont été congelés à $-35^{\circ} \mathrm{C}$ dès la récolte, stockés a $-15^{\circ} \mathrm{C}$, puis lyophylisés et broyés à la grille de $1,5 \mathrm{~mm}$ pour le passage en sachets. Cependant, les 9 fourrages du printemps 1985 ont été broyés à la grille de 0,8 $\mathrm{mm}$, et parmi eux, les 7 graminées ont été congelées dans l'azote liquide. La dégradabilité des fourrages verts est peu affectée par ces modifications de conditionnement (Le Goffe, 1991).

\section{Analyses chimiques}

La teneur en matière sèche (MS) des fourrages frais a été déterminée par passage à l'étuve à $80^{\circ} \mathrm{C}$ pendant $48 \mathrm{~h}$. Les teneurs en matières azotées totales (MAT), NDF (neutral detergent fiber: Van Soest et Wine, 1967), ADF (acid detergent fiber : Van Soest, 1963) et lignine (attaque H2 SO4 : Van Soest, 1963), azote (N) du 
NDF et de l'ADF ont été dosées sur des souséchantillons broyés à la grille de $0,8 \mathrm{~mm}$ (rebroyage ou broyage direct). L'ADF a été obtenu par attaque directe au détergent acide, en revanche la lignine a été dosée après extraction préalable au détergent neutre. La méthode de Van Soest a été automatisée sur Fibertect; les résidus sont déminéralisés.

\section{Mesures de dégradabilité}

Les solubilités $(S)$ et fermentescibilités in vitro $(\mathrm{F})$ de $\mathrm{N}$ ont été déterminées (Vérité et Demarquilly, 1978).

La mesure de la dégradation de MS et $\mathrm{N}$ en sachets de nylon, incubés $2,4,7,10,24$ et $48 \mathrm{~h}$ dans le rumen, a été répétée sur 2 vaches pendant 3 j successifs. Les dosages d'azote ont été effectués après regroupement, à chaque durée d'incubation, des résidus de sachets sur les 3 j d'une même vache. La méthode décrite par Michalet-Doreau et al (1987) a été utilisée avec quelques modifications. Les vaches reçoivent une ration joumalière de $8 \mathrm{~kg}$ de foin de trèfle violet $(16 \% \mathrm{MAT})$ complémenté en minéraux. Les variations de l'activité microbienne ruminale sont prises en compte par ajustement spécifique des cinétiques de dégradation de MS et $\mathrm{N}$ à une méme valeur de dégradation de MS d'un aliment de référence (luzerne déshydratée : $51 \%$ à $10 \mathrm{~h}$ ), comme chez Vérité et al (1989). La relation d'ajustement de la dégradabilité théorique de $\mathrm{N}$ est ainsi : DTN ajustée = DTN observée + 0,43 ( 51 - dégr MS luz $10 \mathrm{~h}$ observée). Après modélisation des cinétiques de dégradation, en moyenne par fourrage, selon le modèle monomoléculaire $(\mathrm{d} / t]=a+b\left[1-\mathrm{e}^{-\mathrm{ct}]}\right]$ ), les dégradabilités théoriques de MS (DTMS) et N (DTN) sont calculées selon la formule établie par Orskov et Mc Donald (1979) : $\mathrm{D} T=a+b c /(c+k)$, avec $k=0,06 \mathrm{~h}^{-1}$.

Les paramètres $a, b$ et $c$ représentent respectivement les fractions immédiatement dégradées et lentement dégradables, et le taux horaire de dégradation de $b$. Les paramètres $a+b$ et $100-(a+b)$ correspondent respectivement aux fractions potentiellement dégradables et indégradables. Si l'on admet l'existence d'une parenté entre fractions indégradables dans le rumen et indigestibles dans l'intestin grêle, le paramètre $(a+b)-D T$ donne alors un indice de la fraction non dégradée dans le rumen et diges- tible dans l'intestin grêle; pour l'azote, il a été utilisé sous le nom de fraction azotée alimentaire digestible.

Parallèlement, les MAT théoriquement non dégradables dans le rumen ( $\mathrm{g} / \mathrm{kg} \mathrm{MS}$ ) peuvent se diviser en MAT indégradables et MAT potentiellement dégradables; ces demières constituent peut-être un indice des MAT non dégradables susceptibles d'être digérées dans l'intestin, c'est-à-dire un indice de la teneur en PDIA des fourrages verts. Cette distinction a permis de déboucher sur un indice de la digestibilité dars l'intestin des MAT non dégradables dans le rumen : $I=(a+b-\mathrm{D}) /(100-\mathrm{D} T)$.

Le rapport $N$ dégradé/MS dégradée a été calculé jusqu'à $4 \mathrm{~h}$ et entre 4 et $10 \mathrm{~h}$ de digestion. Ces 2 ratios ont été reliès à l'efficacité de la synthèse microbienne; Vérité et al (1987) ont montré que la captation optimale de $\mathrm{N}$ par les microbes se situait à $25 \mathrm{~g} / \mathrm{kg}$ de $M O$ fermentescible chez la vache, soit environ $23 \mathrm{~g} /$ kg de MS fermentée pour une teneur en MO de $90 \%$ de MS chez les fourrages verts.

\section{Analyses statistiques}

L'analyse de variance du schéma factoriel espèce $x$ saison a été effectuée selon la procédure GLM du logiciel SAS (1988); il n'y a pas de répétitions. Les moyennes, ajustées pour les données manquantes, sont comparées par le test des ranges multiples de Duncan.

\section{RÉSULTATS}

\section{Composition chimique}

Quelle que soit la saison, les teneurs en MAT de la luzerne et surtout du trèfle violet sont plus faibles que celles des graminées. La teneur en MAT est plus faible en juillet qu'aux autres saisons (tableau II).

Les ray-grass sont moins riches en NDF, ADF et lignine que les fétuques et dactyles, les 3 fractions pariétales évoluant parallèlement chez ces espèces. En re- 
Tableau II. Influence de l'espèce et de la saison sur la composition chimique des fourrages verts.

\begin{tabular}{|c|c|c|c|c|c|c|c|}
\hline Espèce et saison & $\begin{array}{l}M S^{1} \\
(\%)\end{array}$ & $\begin{array}{l}\text { MAT } 2 \\
(\% m s)\end{array}$ & $\begin{array}{l}\text { NDF } \\
\text { (\%ms) }\end{array}$ & $\begin{array}{l}A D F \\
(\% m s)\end{array}$ & $\begin{array}{l}\text { Lignine } \\
(\% m s)\end{array}$ & $\begin{array}{l}N-N D F^{3} \\
\text { (\%nt) }\end{array}$ & $\begin{array}{l}N-A D F^{4} \\
(\% n t)\end{array}$ \\
\hline Ray-grass italien & $20,2^{a}$ & 22,6 a & 42,6 ab & 20,6 a & $1,8^{a}$ & 18,9 ac & $1,8^{a b}$ \\
\hline Ray-grass hybride & 18,4 a & $23,4^{a}$ & 40,9 ab & $20,4^{a}$ & $1,6 \mathrm{a}$ & 17,9 ac & 1,7 ab \\
\hline Ray-grass anglais Réveille & 19,1 a & $23,3^{a}$ & 38,6 a & $20,2 a^{a}$ & $1,7^{\circ}$ & 14,4 ad & 1,7 ab \\
\hline Ray-grass anglais Vigor & $17,3^{\text {a }}$ & $21,9 a b$ & 41,8 ab & 22,5 ac & 1,7 a & 14,3 ad & $1,3 a$ \\
\hline Fétuque élevée Clarine & $19,1^{\text {a }}$ & $21,9 a b$ & $45,8^{b}$ & 24,4 bc & $1,8^{a}$ & $8,6^{b}$ & 1,6 ab \\
\hline Fétuque élevée Lubrette & 19,1 a & $21,1 \mathrm{abc}$ & $46,3^{b}$ & 23,9 abc & $1,8^{a}$ & 12,3 ab & 1,7 ab \\
\hline Dactyle & $19,4^{a}$ & 23,1 a & $47,8^{b}$ & 24,6 bc & $2,4^{a}$ & $20,5^{c}$ & $2,0^{b}$ \\
\hline Trèfle violet & $13,7^{b}$ & $17,8^{c}$ & 41,7 ab & $26,5^{b}$ & $3,8 \mathrm{~b}$ & 16,7 ac & $3,8^{c} \mathrm{c}$ \\
\hline Luzeme & $17,5^{a}$ & $18,9 b c$ & 42,4 ab & $31,7^{d}$ & $6,0^{c}$ & 10,6 bd & $4,2^{c}$ \\
\hline Avril-mai 1985 & $15,7^{a}$ & $23,2^{a}$ & $40,4^{a}$ & $22,5^{a}$ & $2,2^{\mathrm{a}}$ & $16,3^{a}$ & 1,9 \\
\hline Mai-juin 1986 & $14,0^{b}$ & $21,0^{b c}$ & $44,8^{b}$ & $26,3^{c}$ & 2,4 a & $12,1^{b}$ & 2,0 \\
\hline Juillet 1985 & $19,9^{c}$ & $19,3^{c}$ & 43,5 ab & $26,3^{c}$ & $3,3^{b}$ & 13,8 ab & 2,4 \\
\hline Septembre 1985 & $22,6^{d}$ & $21,7 \mathrm{ab}$ & $46,4^{b}$ & $23,8^{a}$ & 2,9 b & 17,5 a & 2,2 \\
\hline Écart type résiduel 5 & 1,6 & 1,9 & 3,6 & 2,1 & 1,2 & 3,6 & 0,7 \\
\hline
\end{tabular}

1 Matière sèche; ${ }^{2}$ matières azotées totales; ${ }^{3}$ azote lié au NDF, en pourcentage de l'azote total; ${ }^{4}$ azote lié à l'ADF, en pourcentage de l'azote total; ${ }^{5}$ de l'analyse de variance selon modèle : $Y=$ moyenne + espéce + saison, + résjduelle; $a, b, c, d$ : dans une colonne et par facteur, moyennes ajustées par le modèle significativement différentes $(P<0,05)$.

vanche, à même teneur en NDF que les ray-grass, le trèfle violet et surtout la luzerne sont beaucoup plus riches en ADF et en lignine. Les teneurs en NDF et ADF sont significativement plus faibles en avrilmai 1985 qu'en mai-juin 1986 et qu'aux autres saisons. Ceci est particulièrement marqué pour la teneur en ADF chez les graminées et a pour origine l'exploitation très précoce au printemps 1985 (stade feuillu du premier cycle contre stade épi à $10 \mathrm{~cm}$ au printemps 1986). La teneur en lignine est significativement plus faible au printemps qu'en juillet et en septembre.

La proportion de l'azote total liée au NDF est minimale chez la fétuque Clarine $(8,6 \%)$ et la luzerne $(10,6 \%)$ et maximale chez le dactyle $(20,5 \%)$. La proportion de l'azote total liée à l'ADF est plus élevée chez les légumineuses (4\%) que chez les graminées (1,5 à $2 \%$ ). Les proportions de l'azote total liées aux parois varient peu avec la saison.

\section{Dégradabilité de la matière sèche}

Les légumineuses sont dégradées plus rapidement que les graminées. Néanmoins, la DTMS de la luzerne est inférieure à celle des autres espèces, car sa fraction lentement dégradable est plus faible, par suite d'une proportion de MS indégradable maximale (tableau III). À l'opposé, la DTMS élevée des ray-grass résulte de l'importance de leurs fractions immédiatement et lentement dégradables. Les fétuques et le dactyle ont une DTMS intermé- 
Tableau III. Influence de l'espèce et de la saison sur la dégradabilité de la matière sèche des fourrages verts en sachets (en pourcentage).

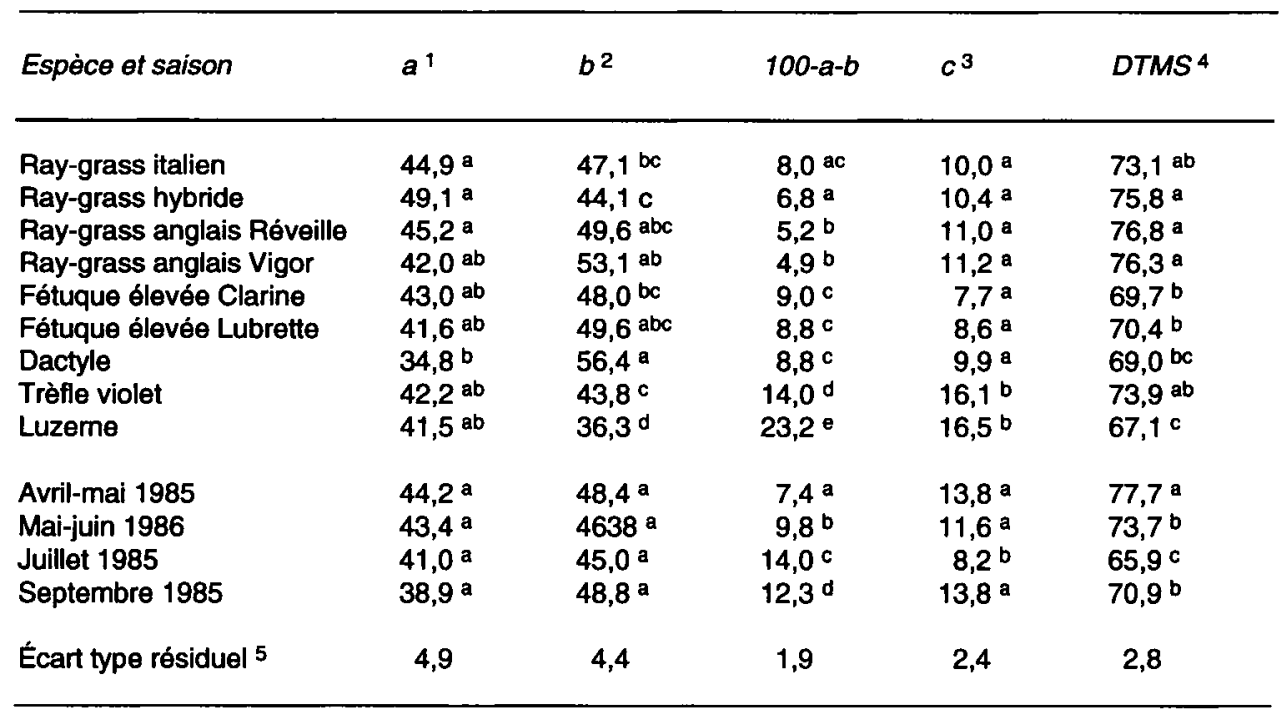

1 Fraction immédiatement dégradée; ${ }^{2}$ fraction lentement dégradable; ${ }^{3}$ taux horaire de dégradation de b; ${ }^{4}$ dégradabilité théorique; $k=0,06 \mathrm{~h}^{-1} ;{ }^{5}$ de l'analyse de variance selon modèle : $Y=$ moyenne + espéce + saison + résiduelle; $a, b, c, d, e:$ dans une colonne et par facteur, moyennes ajustées par le modèle significativement différentes $(P<$ $0,05)$.

diaire; elle est à relier chez les premières à une faible vitesse de dégradation, et chez le second à une fraction immédiatement dégradée faible. Le classement des espèces ne varie pas nettement avec la saison. L'évolution saisonnière du taux horaire de dégradation, et à un moindre degré, celle de la dégradabilité potentielle, expliquent les différences de DTMS entre saisons. La DTMS est maximale en avrilmai 1985, diminue significativement en mai-juin 1986, puis plus fortement en juillet et remonte en septembre.

\section{Dégradabilité de l'azote}

$S$ et $F$ sont assez étroitement liées et leurs valeurs individuelles sont relativement proches $\left(F=0,85 S+5,8 ; s=4,9 ; R^{2}=\right.$
0,$62 ; N=31$ ). En moyenne, $S$ et $F$ sont faibles chez le trèfle violet et le dactyle, et élevées pour la majorité des ray-grass; l'azote de la luzerne est également très soluble, mais pour cette espèce et les fétuques le rapport $F / S$ est faible (tableau IV). Les valeurs moyennes de $S$ et $F$ et leurs variations avec la saison sont très proches. $S$ et $F$ sont maximales en avrilmai 1985, diminuent en mai-juin 1986 et restent stables en juillet, puis diminuent à nouveau en septembre. Le classement des espèces varie peu selon la saison, cependant $S$ remonte en septembre chez le dactyle surtout, et la luzerne.

La DTN varie peu selon l'espèce (de 80 à $86 \%$ ); le dactyle surtout et le trèfle violet ont cependant une DTN significativement inférieure à celle des autres espèces (tableau IV). Cette faible variation est due à 
Tableau IV. Influence de l'espèce et de la saison sur la dégradabilité de l'azote des fourrages verts in vitro et en sachets (en pourcentage).

\begin{tabular}{|c|c|c|c|c|c|c|c|}
\hline Espèce et saison & $S^{1}$ & $F^{2}$ & $a^{3}$ & $b^{4}$ & $100-a-b$ & $c^{5}$ & $D T N^{6}$ \\
\hline $\begin{array}{l}\text { Ray-grass italien } \\
\text { Ray-grass hybride } \\
\text { Ray-grass anglais Réveille } \\
\text { Ray-grass anglais Vigor } \\
\text { Fétuque élevée Clarine } \\
\text { Fétuque élevée Lubrette } \\
\text { Dactyle } \\
\text { Trèfle violet } \\
\text { Luzerne }\end{array}$ & $\begin{array}{l}56,0^{\text {a }} \\
55,0^{\text {ab }} \\
51,2 \text { bcd } \\
47,7 \text { de } \\
49,5 \text { cde } \\
50,2^{\text {cde }} \\
46,0^{\text {ef }} \\
42,4^{f} \\
53,5 \text { abc }\end{array}$ & $\begin{array}{l}57,5^{a} \\
56,4^{a} \\
53,9^{a} \\
44,8^{b} \\
44,4^{b} \\
45,4^{b} \\
42,5^{b} \\
46,8^{b} \\
47,6^{b}\end{array}$ & $\begin{array}{l}55,4 \text { ab } \\
57,2 \text { ab } \\
52,0 \text { abc } \\
51,4 \text { abc } \\
62,5 \text { a } \\
56,8 \text { ab } \\
44,3 \text { bc } \\
41,7 \mathrm{c} \\
55,6 \text { ab }\end{array}$ & $\begin{array}{l}38,7^{\mathrm{b}} \\
37,6^{\mathrm{b}} \\
45,1^{\mathrm{ab}} \\
44,8^{\mathrm{ab}} \\
33,3^{\mathrm{b}} \\
38,4^{\mathrm{b}} \\
53,0^{\mathrm{a}} \\
51,3^{\mathrm{a}} \\
35,6^{\mathrm{b}}\end{array}$ & $\begin{array}{l}5,9 \mathrm{bc} \\
5,2 \mathrm{abc} \\
2,9 \mathrm{a} \\
3,8 \mathrm{ab} \\
4,2 \mathrm{abc} \\
4,8 \mathrm{abc} \\
2,7 \mathrm{abc} \\
7,0 \mathrm{~cd} \\
8,8 \mathrm{~d}\end{array}$ & $\begin{array}{l}16,9^{\mathrm{b}} \\
19,3^{\mathrm{ab}} \\
16,8^{\mathrm{ab}} \\
20,6^{\mathrm{ab}} \\
12,4^{\mathrm{b}} \\
15,7^{\mathrm{b}} \\
13,4^{\mathrm{b}} \\
21,3^{\mathrm{ab}} \\
27,5^{\mathrm{a}}\end{array}$ & $\begin{array}{l}83,7 \text { ab } \\
85,7 \text { a } \\
85,2 \text { a } \\
85,8 \text { a } \\
84,9 \text { a } \\
89,9 \text { ab } \\
79,6 \text { b } \\
81,6 \text { ab } \\
84,9 \text { a }\end{array}$ \\
\hline $\begin{array}{l}\text { Avril-mai } 1985 \\
\text { Mai-juin } 1986 \\
\text { Juillet } 1985 \\
\text { Septembre } 1985\end{array}$ & $\begin{array}{l}57,3^{a} \\
48,9^{b} \\
48,5^{b} \\
40,6^{c}\end{array}$ & $\begin{array}{l}57,4^{a} \\
43,8^{b} \\
45,1^{b} \\
40,3^{c}\end{array}$ & $\begin{array}{l}54,6^{\text {a }} \\
64,0^{\text {b }} \\
50,7^{\text {a }} \\
42,0^{c}\end{array}$ & $\begin{array}{l}41,2^{a} \\
31,4^{b} \\
42,3^{a} \\
53,5^{c}\end{array}$ & $\begin{array}{l}4,2^{a} \\
4,6^{a} \\
7,0^{b} \\
4,5^{a}\end{array}$ & $\begin{array}{l}20,4 \\
18,3 \\
16,1 \\
19,8\end{array}$ & $\begin{array}{l}86,4^{a} \\
87,6^{a} \\
80,1^{b} \\
82,0^{b}\end{array}$ \\
\hline Écart type résiduel 7 & 2,6 & 5,9 & 6,9 & 7,0 & 1,4 & 5,4 & 2,5 \\
\hline
\end{tabular}

1 Solubilité; 2 fermentescibilité in vitro, ${ }^{3}$ fraction immédiatement dégradée; ${ }^{4}$ fraction lentement dégradable; ${ }^{5}$ taux horaire de dégradation de $b ;{ }^{6}$ dégradabilité théorique; $k=0,06 \mathrm{~h}^{-1} ;{ }^{7}$ de l'analyse de variance selon modèle : $Y=$ moyenne + espèce + saison + résiduelle; $a, b, c, d, e, f$; dans une colonne et par facteur, moyennes ajustées par le modèle significativement différentes $(P<0,05)$.

une compensation entre les paramètres de la cinétique; en effet, les courbes de dégradation de $\mathrm{N}$ peuvent être assez différentes suivant les espèces (fig 1. et tableau IV). Les légumineuses sont caractérisées par une fraction indégradable et une vitesse de dégradation plus élevée que celles des graminées. La plus faible DTN du dactyle et du trèfle violet s'explique en partie par une fraction immédiatement dégradée très faible, ce qui les oppose à la luzerne et aux autres graminées. La fraction b du dactyle est importante et se dégrade avec une faible vitesse, ce qui conduit à une fraction azotée alimentaire digestible maximale; c'est l'inverse chez la luzerne (fig 2).

La forme de la courbe de dégradation de $\mathrm{N}$ varie également selon la saison. La fraction immédiatement dégradée est net- tement plus faible en septembre qu'en juillet ou au printemps, surtout en 1986. La vitesse de dégradation diminue en juillet, mais non significativement. La proportion de $\mathrm{N}$ indégradable est significativement plus élevée en juillet qu'aux autres saisons. L'évolution saisonnière de la cinétique de dégradation se répercute sur la DTN qui est maximale au printemps, diminue en juillet et remonte légèrement en septembre. Cependant, la remontée de septembre intervient surtout pour le trèfle violet et le dactyle, et moins pour les autres espèces (fig 3). Par ailleurs, la DTN du trèfle violet au printemps semble diminuée par une récolte tardive. Chez la plupart des espèces, la DTN diminue lorsque la température moyenne de pousse augmente $\left(\mathrm{DTN}=94,7-0,85 T ; s=2,9 ; R^{2}=\right.$ 0,$56 ; n=31$ ). 


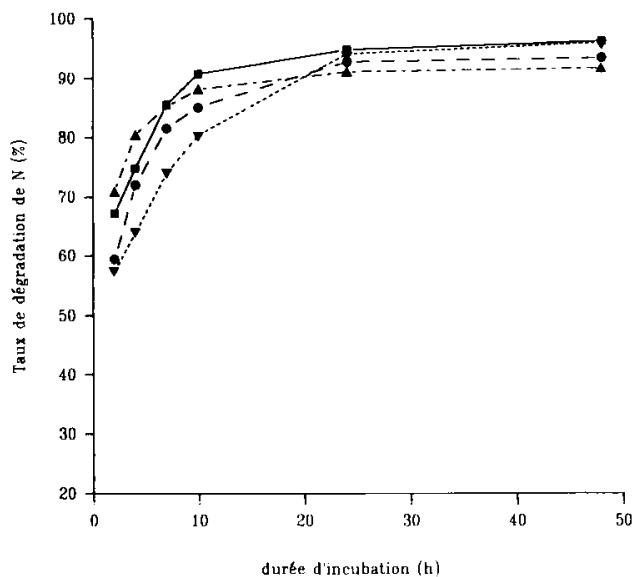

Fig 1. Influence de l'espèce sur la cinétique de dégradation de l'azote en sachets. I ray-grass; $\boldsymbol{\nabla}$ dactyle; - trèfle violet; $\boldsymbol{\Delta}$ luzerne.
L'indice de la teneur en PDIA varie avec l'espèce entre 12 (luzerne) et $41 \mathrm{~g} / \mathrm{kg}$ de MS (dactyle); il est plus élevé en été qu'au printemps (fig 4). L'indice de la digestibilité des MAT non dégradables varie de $42 \%$ (luzerne) à $87 \%$ (dactyle et ray-grass anglais Vigor); les autres espèces sont autour de $70 \%$ (fig 5). L'indice de digestibilité ne varie pas nettement avec la saison, il est plus élevé en septembre.

En moyenne, au début de l'incubation, la luzerne surtout et les graminées s'appauvrissent davantage en azote que le trèfle violet (fig 6); cela correspond à un ratio $N$ dégradé/MS dégradée jusqu'à $4 \mathrm{~h}$ plus faible chez le trèfle violet $(30 \mathrm{~g} / \mathrm{kg}$ ) que pour les autres espèces (41 à $48 \mathrm{~g}$ / $\mathrm{kg}$ ). Ce ratio varie peu avec la saison, et ses valeurs individuelles sont toujours supérieures à $25 \mathrm{~g} / \mathrm{kg}$ chez le trèfle violet et à $35 \mathrm{~g} / \mathrm{kg}$ pour les autres espèces; il présente une liaison positive étroite avec la teneur en MAT (N/MS 0-4 $\mathrm{h}=8,0+1,64$

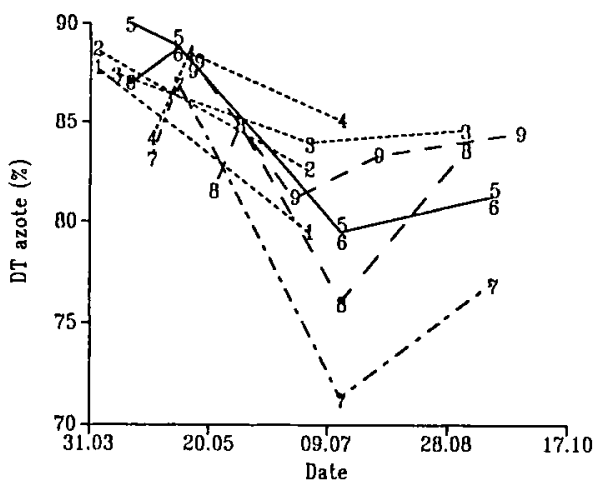

Fig 3. Evolution selon la saison de la dégradabilité théorique de l'azote de 9 fourrages verts $(k=$ $\left.0,06 h^{-1}\right) .1$ : ray-grass italien; 2 : ray-grass hybride; 3 : ray-grass anglais Réveile; 4 : raygrass anglais Vigor; 5 : fétuque élevée Clarine; 6 : fétuque élevée Lubrette; $7:$ dactyle; 8 : trèfle violet; 9 : luzerne.
Fig 2. Influence de l'espèce et de la saison sur la fraction azotée lentement dégradable, son taux horaire de dégradation, et la fraction azotée alimentaire digestible. rgr: ray-grass; fet : fétuque; dac : dactyle; trv : trètle violet; luz : luzerne; P1 : printemps 1985; P2 : printemps 1986; J1 : juillet 1985; S1 : septembre 1985. 


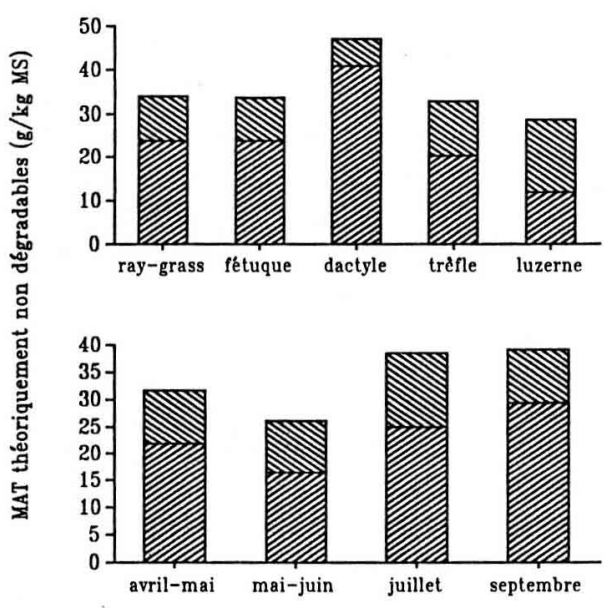

Fig 4. Influence de l'espèce et de la saison sur la répartition des matières azotées théoriquement non dégradables $\left(k=0,06 \mathrm{~h}^{-1}\right)$.

7 potentiellement dégradables (indice PDIA); indégradables.

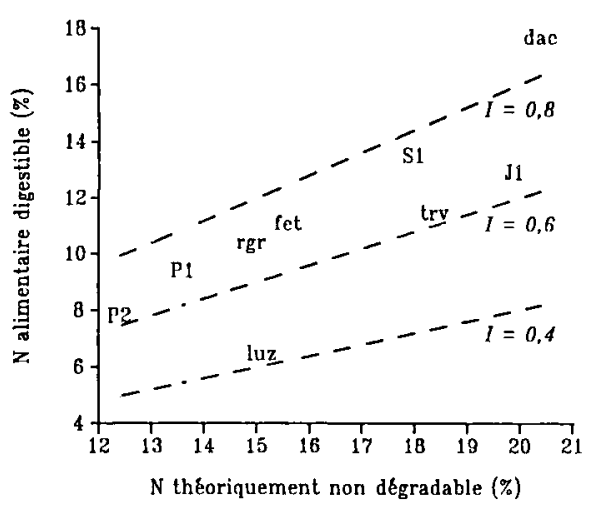

Fig 5. Influence de l'espèce et de la saison sur l'estimation de la digestibilité dans l'intestin de l'azote non dégradable dans le rumen $(\mathbf{k}=$ $\left.0,06 \mathrm{~h}^{-1}\right)$. I: indice de digestibilité; rgr : ray-grass; fet : fétuque; dac : dactyle; trv : trèfle violet; luz: luzeme; P1 : printemps 1985; P2 : printemps 1986; J1 : juillet 1985; S1 : septembre 1985.
MAT; $\left.s=3,7 ; R^{2}=0,64 ; n=31\right)$. Entre 4 et $10 \mathrm{~h}$, le ratio $\mathrm{N}$ dégradé/MS dégradée peut atteindre des valeurs inférieures à 23 $\mathrm{g} / \mathrm{kg}$ à certaines saisons, chez la luzerne surtout (printemps 86 et juin 85 ) et chez les fétuques (fig 7).

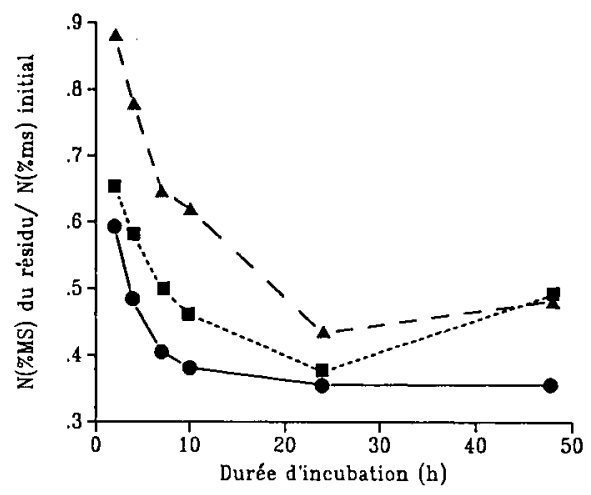

Fig 6. Influence de l'espèce sur l'évolution de la teneur en azote des résidus de sachets au cours de l'incubation. $\square$ fétuque élevée; $\boldsymbol{\Delta}$ trèfle violet; @ luzerne.

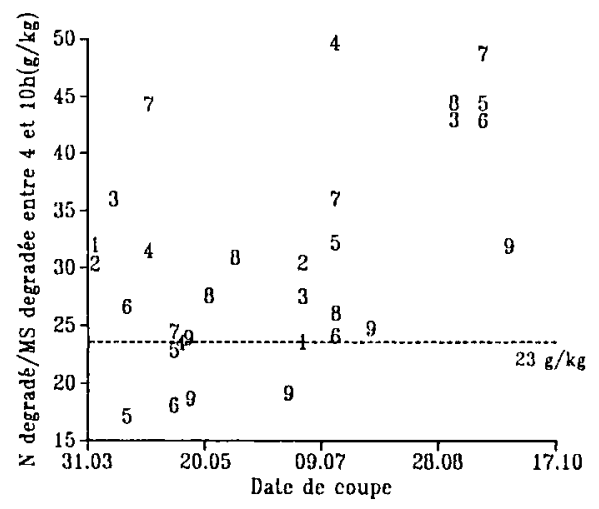

Fig 7. Évolution selon la saison du rapport azote dégradé/matière sèche dégradée entre 4 et $10 \mathrm{~h}$ chez 9 fourrages verts. 1 : ray-grass italien; 2 : ray-grass hybride; 3 : ray-grass anglais Réveille; 4 : ray-grass anglais Vigor; 5 : fétuque élevée Clarine; 6 : fétuque élevée Lubrette; 7 : dactyle; 8 : trèfle violet; 9 : luzerne. 


\section{DISCUSSION}

\section{La famille et l'espèce}

Par rapport à celles des graminées, les cinétiques de dégradation de $\mathrm{MS}$ et $\mathrm{N}$ des légumineuses et surtout de la luzerne sont caractérisées par des valeurs élevées : 1) de la vitesse de dégradation de la fraction lentement dégradable; 2 ) de la fraction indégradable. Des observations similaires sur la vitesse de dégradation en sachets avaient été faites par Osibe et al (1987), Van Eys et Reid (1987), Vik-Mo (1989). Demarquilly et Jarrige (1973) avaient déjà signalé la digestion plus rapide des légumineuses dans le rumen à même digestibilité, et la digestibilité plus faible de la luzerne à même stade. La fraction azotée indégradable plus élevée chez les légumineuses est à rapprocher de leur proportion de $\mathrm{N}$ liée à l'ADF supérieure à celle des graminées. Cependant, sur les autres critères de dégradabilité de $\mathbf{N}$, des différences existent davantage entre espèces qu'entre familles. Trois espèces ont des caractéristiques plus particulières : la luzerne, le trèfle violet et le dactyle.

Chez la luzerne, les fractions azotées rapidement dégradables $(S, F$, fraction a) ont été généralement importantes, comme l'avaient montré précédemment Osibe et al (1987), Abdalla et al (1988a), Sanderson et Wedin (1989). Cela est à mettre en relation avec les proportions de $\mathrm{N}$ non protéique et d'acides aminés libres dans l'azote totale, plus élevées chez la luzerne que chez les autres fourrages (Demarquilly et al, 1981; Mangan, 1982). Les différences de DTN entre la luzerne et les graminées obtenues par Osibe et al (1987) sont plus importantes et plus systématiques que les nôtres. Mais dans cette étude, les légumineuses étaient exploitées plus précocement que les graminées, et les fourrages étaient séchés à l'étuve, méthode qui augmente les écarts de DTN par rapport à la Iyophilisation (Kamoun et Thewis, 1989).

Le trèfle violet, à l'opposé de la luzerne, a été caractérisé par des fractions azotées rapidement dégradables plus faibles que celles des autres espèces, ce qu'avaient déjà obtenu Syrjälä-Qvist et al (1984), Osibe et al (1987), Van Eys et Reid (1987), Vik-Mo (1989), Sanderson et Wedin (1989). Parallèlement, le trèfle violet ferait partie des fourrages possédant la plus faible proportion d'acides aminés libres dans l'azote total (Mangan, 1982). Ces proportions d'azote soluble et immédiatement dégradé faibles ont été compensées par une vitesse de dégradation plus élevée, de sorte que la DT du trèfle violet n'a pas été significativement différente de celles des ray-grass et de la luzerne. Van Eys et Reid (1987) et Vik-Mo (1989) sur ensilage n'ont pas constaté de différence entre la DT du trèfle violet et celles des graminées.

Chez le dactyle, les fractions azotées rapidement dégradables, la vitesse de dégradation et donc la DTN ont toujours été faibles, comme l'avaient constaté antérieurement Glenn et al (1985) et, mais moins nettement, Abdalla et al (1988b) et Osibe et al (1989). Parallèlement, la proportion de $\mathrm{N}$ soluble dans le détergent neutre a été inférieure à celle de toutes les autres espèces. Ces observations sont cohérentes avec les faibles proportions de $\mathrm{N}$ sous forme non protéique ou d'acides aminés libres, déjà observées chez le dactyle (Glenn et al, 1985; Mangan, 1982). Par ailleurs, le dactyle a été plus riche en NDF, $A D F$ et lignine que les autres graminées (voir aussi Abdalla et al, 1988b). Cette plus grande proportion de parois, probablement plus lignifiées, en diminuant l'accessibilité des contenus cellulaires, explique peutêtre également la moindre dégradabilité du dactyle. 
L'indice de la teneur en PDIA du dactyle est supérieur à celui des autres graminées et plus encore à celui de la luzerne. Pour une vache ingérant de 12 à $15 \mathrm{~kg}$ de MS, l'écart maximal représenterait 350 à $450 \mathrm{~g}$ de PDIA, soit une différence importante d'apport azoté. Cependant, les écarts de DT et d'indice de PDIA entre fourrages pourraient être modifiés si la vitesse de transit diffère selon la nature du fourrage. Ainsi, les légumineuses transitent probablement plus vite que les graminées, et les ray-grass plus rapidement que les fétuques et les dactyles, comme le suggèrent nos résultats de dégradation de la MS, et aussi Demarquilly et al (1981), Lindberg (1985), Ould-Bah (1989). L'indice de la teneur en PDIA des légumineuses serait donc sousestimé par rapport à celui des graminées.

\section{La saison}

La vitesse de dégradation, la dégradabilité potentielle et la DT de $N$ en sachets, ainsi que la proportion de $\mathrm{N}$ soluble dans le détergent acide, ont présenté une évolution saisonnière caractéristique : maximum au printemps, baisse en juillet et remontée en septembre. Cette évolution se dessinait déjà dans le regroupement des rares études de dégradabilité consacrées à la saison, bien qu'elles aient été réalisées à des périodes différentes de l'année sur un nombre restreint d'espèces, et avec des méthodes très diverses (azote du NDF, solubilité, dégradation enzymatique, dégradation in vitro, méthode des sachets : Aman et Lindgren, 1983; Moré et Simon, 1985; Hafley et al, 1987; Abdalla et al, 1988b et c; Tamminga et al, 1991; Le Goffe et Vérité, 1992). En revanche, les critères représentant la fraction rapidement dégradable (azote soluble dans le détergent neutre, S, F, fraction a) ont eu une évolution différente ou aléatoire, en contradiction avec les données bibliographiques.
In vivo, Beever et al (1986) ont montré que la dégradabilité de l'azote (N15) était légèrement mais significativement plus faible en juillet qu'au premier cycle ou en septembre, et Vérité et al (1984) obtiennent en automne une concentration en ammoniac ruminal qui suggère une dégradabilité plus élevée qu'aux autres saisons.

L'évolution saisonnière de la DTN est à rapprocher de celle de la digestibilité de la matière organique qui serait principalement sous l'influence de la température (Demarquilly, 1982b). Les températures élevées sont connues pour stimuler la croissance de la plante (diminution du pourcentage de feuilles) et augmenter la lignification des parois (Deinum, 1984). L'augmentation de la teneur en lignine avec la température, alors que les constituants pariétaux ne varient pas, a déjà été signalée pour les fourrages tropicaux (Demarquilly, 1982b). Le minimum estival de DTN pourrait partiellement provenir de la moindre accessibilité des contenus cellulaires par suite de la lignification accrue des parois, comme le pensent Abdalla et al (1988b). Une autre explication partielle de l'élévation de la dégradabilité de l'azote au printemps et à l'automne réside peut être dans l'accumulation des formes azotées solubles (notamment les nitrates), à laquelle conduirait le ralentissement de la protéosynthèse associé à la baisse de la température et du rayonnement (Moré et Simon, 1985).

Enfin, comme Abdalla et al (1988b), nous obtenons une estimation des MAT non dégradables dans le rumen susceptibles d'être digérées dans l'intestin plus élevée en été qu'au printemps.

\section{CONCLUSION}

L'espèce et la saison influencent modérément la dégradabilité de l'azote en sachets 
de nylon. La DTN est toujours élevée, et les pertes azotées en début de digestion doivent être très importantes chez les ruminants consommant des fourrages verts jeunes. Toutefois, la fraction azotée rapidement dégradable varie notablement selon l'espèce, en relation avec des natures d'azote différentes (proportion d'azote non protéique notamment). En agissant sur la lignification des parois, la température déterminerait en partie l'évolution saisonnière de la DT.

Les possibilité de manipuler la dégradabilité de l'azote des fourrages verts en choisissant l'espèce semblent limitées; mais notre étude ne comportait pas de plantes riches en tannins telles les lotiers et les sainfoins, dont les protéines seraient peu dégradables. L'amélioration de la valeur azotée des fourrages verts passe peut-être également par la sélection de plantes à faible proportion d'azote non protéique et aux protéines peu dégradables dans le rumen.

\section{REMERCIEMENTS}

Nous adressons nos sincères remerciements à l'ensemble du personnel (installations expérimentales, laboratoire et secrétariat) de la station INRA de recherches sur la vache laitière.

\section{RÉFÉRENCES}

Abdalla HO, Fox DG, Van Soest PJ (1988a) An evaluation of methods for preserving fresh forage samples before protein fraction determination. J Anim Sci 66, 2646-2649

Abdalla HO, Fox DG, Seaney RR (1988b) Protein distribution in four cool-season grass varieties alone or in combination with trefoil. J Anim Sci 66, 2325-2329

Abdalla HO, Fox DG, Seaney RR (1988c) Variation in protein and fiber fractions in pasture during the grazing season. $J$ Anim Sci 66 , 2663-2667

Aman P, Lindgren E (1983) Chemical composition and in vitro degradability of individual constituents of six Swedish grasses harvested at different stages of maturity. Swed $J$ Agric Res 13, 221-227

Beever DE, Dhanoa MS, Losada HR, Evans RT, Cammel SB, France J (1986) The effect of forage species and stage of harvest on the processes of digestion occuring in the rumen of cattle. Br J Nutr 56, 439-454

Deinum B (1984) Chemical composition and nutritive value of herbage in relation to climate. In: Proceedings of the tenth general meeting of the European Grassland Federation, As Norway (Riley H, Skjelvag AO, eds). The Norwegian State Agricultural Research Stations, Moervegen, 338-350

Demarquilly $C$ (1982a) Valeur alimentaire des fourrages. In: Forum des fourrages de l'ouest. ITCF, Rennes, 95-101

Demarquilly C (1982b) Influence des facteurs climatiques sur la composition et la valeur nutritive de l'herbe. In: Actions du climat sur l'animal au pâturage. INRA, Versailles, 49-63

Demarquilly C, Grenet E, Andrieu J (1981) Les constituants azotés des fourrages et la prévision de la valeur azotée des fourrages. In: Prévision de la valeur nutritive des aliments des ruminants. INRA, Versailles, 129-154

Demarquilly C, Jarrige R (1973) The comparative nutritive value of grasses and legumes. Växtodling 28, 33-41

Demarquilly C, Weiss $P$ (1970) Valeur alimentaire des fourrages verts. Fourrages $42,3-22$

Glenn BP, Ely DG, Glenn S, Douglas LW, Bull LS, Bush LP (1985) Effects of ammonium nitrate and potassium sulfate fertilization on rates of ruminal in situ disappearance of tall fescue and orchard grass nitrogen and sulfur. Can J Anim Sci 65, 631-646

Hafley J, Nipper WA, Craig WM, Adkinson RW, Achacoso AS (1987) Effect of growth stage and fertilization on crude protein and in vitro protein degradation of cool-season annual forages. J Dairy Sci 70, 2550-2559

Kamoun $M$, Thewis A (1989) Influence du mode de conditionnement d'un fourrage vert sur sa composition chimique, la digestibilité in vitro de la matière organique et la dégradabilité in 
sacco de l'azote dans le rumen. Reprod Nutr Dév suppl 2, 159s-160s

Le Goffe P (1991) Méthodes d'étude et facteurs de variation de la dégradabilité de l'azote des fourrages verts dans le rumen. Thèse de l'École nationale supérieure agronomique de Rennes, $82 p$

Le Goffe P, Vérité R (1992) Evolution de la dégradabilité de l'azote du ray-grass anglais au cours de la saison de pâturage. Reprod Nutr Dev (Ann Zootech 41, 39-40)

Lindberg JE (1985) Estimation of rumen degradability of feed proteins with the in sacco technique and various in vitro methods: a review. Acta Agric Scand suppl 25, 64-97

Mangan JL (1982) The nitrogenous constituents of fresh forages. In: Forage protein in ruminant animal production (Thomson DJ, Beever DE, Gunn RG, eds). British Soc An Prod, occ publ 6, Haddington 25-40

Michalet-Doreau B, Vérité R, Chapoutot P (1987) Méthodologie de mesure de la dégradabilité in sacco de l'azote des aliments dans le rumen. Bull Tech CRZV Theix INAA 69, 5-7

Moré E, Simon JC (1985) Évolution au cours de l'année des teneurs en azote soluble du raygrass d'ltalie de printemps. Fourrages 103, 79-94

Orskov ER, Mc Donald I (1979) The estimation of protein degradability in the rumen from incubation measurements weighted according to rate of passage. J Agric Sci Camb 92, 499-503

Osibe A, Ogawa M, Masubichi T (1987) The degradability of crude forage protein in the rumen of sheep. I. A comparison of leguminous and graminaceous crude protein degradability. Bull Natl Grass/ Res Inst 37, 58-63

Ould-Bah MY (1989) Adaptation de la technique in sacco à l'étude de la dégradation dans le rumen de l'azote des fourrages et application à l'étude des fourrages verts et conservés. Thèse Université de Montpellier, $186 \mathrm{p}$

Sanderson MA, Wedin WF (1989) Nitrogen in the detergent fiber fractions of temperate legumes and grasses. Grass Forage Sci 44, $159-168$
SAS (1988) SAS/STAT guide for personal computers, version 6. SAS Inst Inc, Cary, NC, USA

Syrjälä-Qvist L, Pekkarinen E, Setälä J, Kangasmäki $T$ (1984) Effect of redclover/timothy ratio on the protein feeding value and the quality of silage. J Agric Sci Fin/ 56, 183-192

Tamminga $S$, Ketelaar $R$, Van Vuuren AM (1991) Degradation of $N$ in conserved forages in the rumen of dairy cows. Grass Forage Sci 46, 427-435

Van Eys JE, Reid RL (1987) Ruminal solubility of nitrogen and minerals from fescue and fescue-red clover herbage. J Anim Sci 65, 1101-1112

Van Soest PJ (1963) Use of detergents in the analysis of fibrous feeds. 2. A rapid method for the determination of fiber and lignin. $J$ Assoc Off Anal Chem 46, 829-835

Van Soest PJ, Wine RH (1967) Use of detergents in the analysis of fibrous feeds. Determination of plant cell-wall constituants. $J$ Assoc Off Agric Chem 50, 50-55

Vérité $R$, Demarquilly $C$ (1978) Qualité des matières azotées des aliments pour ruminants. In : La vache laitière. INRA, Versailles, 143157

Vérité R, Michalet-Doreau B, Chapoutot P, Peyraud $\mathrm{JL}$, Poncet $\mathrm{C}$ (1987) Révision du système des protéines digestibles dans l'intestin (PDI). Bull Tech CRVZ Theix INRA 70, 19-34

Vérité R, Rémond $B$, Journet M (1984) Sites of organic matter and protein digestion in lactating cows feed grass from spring to autumn. Can J Anim Sci 64, 328-329

Vérité R, Michalet-Doreau B, Vedeau F, Chapoutot $P$ (1989) Dégradabilité en sachets des matières azotées des aliments concentrés : standardisation de la méthode et variabilités intra- et interlaboratoires. Reprod Nutr Dév suppl 2, 161s-162s

Vik-Mo $L$ (1989) Degradability of forages in sacco. II. Silages of grasses and red clover at two cutting times, with formic acid and without additives. Acta Agric Scand 39, 5364 